\title{
FINITE GROUP SCHEMES OVER FIELDS
}

\author{
RAYMOND T. HOOBLER AND ANDY R. MAGID
}

ABSTRACT. A short proof that commutative group schemes over a field form an abelian category is given.

The category of commutative finite group schemes (equivalently, the category of commutative, cocommutative Hopf algebras with antipode) over a field is abelian [3], [4, Chapter 16]. This note presents an elementary proof of this fact.

We make the following conventions: $k$ will denote a field and $k$-Alg the category of commutative $k$-algebras; unsubscripted tensors are over $k$. Let $k$-FCGS denote the category of covariant functors from $k$-Alg to abelian groups represented by finite dimensional $k$-algebras. The objects of $k$-FCGS are called finite commutative group schemes. For $G$ in $k$-FCGS, $A G$ will denote the $k$-algebra representing $G\left(\right.$ also $G(T)=\operatorname{Hom}_{k \text {-Alg }}(A G, T)$ ). Let $[G: 1]$ be the $k$-dimension of $A G$. By the Yoneda lemma [1, p. 113] (which we will subsequently employ without explicit reference), a morphism $f: G \rightarrow H$ in $k$-FCGS is induced by a unique morphism $A f: A H \rightarrow A G$ in $k$-Alg. A morphism $f: G \rightarrow H$ in $k$-FCGS is free if $A f$ makes $A G$ a free $A H$-module. We say "define $G \rightarrow H$ by $a \rightarrow a$ "' for "let $G \rightarrow H$ be the transformation which, on $T$, sends $G(T)$ to $H(T)$ by sending $a$ to $a^{\prime \prime}$ ".

We refer the reader to [3] for the facts that $k$-FCGS is a self-dual category ("Cartier duality"); that it has fibre products and cofibred coproducts, hence kernels and cokernels, and $A\left(G \times{ }_{H} L\right)=A G \otimes_{A H} A L$; and that $G \rightarrow H$ is an epimorphism in $k$-FCGS if and only if $A H \rightarrow A G$ is injective. The null object 0 of $k$-FCGS is represented by $k$.

To show $k$-FCGS abelian, it suffices to prove, since the category is self dual, epimorphisms are cokernels. This will be done by showing that epimorphisms are free.

Lemma 1. Let $L \rightarrow G$ be the kernel of $G \rightarrow H$. Then $G \times{ }_{H} G \rightarrow G$ (by projection on the first factor) is free of rank $[L: 1]$.

Received by the editors March 22, 1971.

AMS 1970 subject classifications. Primary 14L20; Secondary 16A24, 18 B99.

Key words and phrases. Group scheme, abelian category, duality. 
Proof. Since $L=G \times{ }_{H} 0, L(T) \rightarrow G(T)$ is the kernel of $G(T) \rightarrow H(T)$. Define $d: G \times L \rightarrow G \times{ }_{H} G$ by $(g, l) \rightarrow(g, g l)$. By the above, $d(T)$ is an isomorphism for each $T$, and hence $d$ is an isomorphism. Moreover $d$ commutes with projection on the first factor and hence defines an isomorphism of $A G$ algebras $A d: A G \otimes{ }_{A H} A G \rightarrow A G \otimes A L$. The latter is free of rank $[L: 1]$, whence the result.

LEMMA 2. Let $A$ and $B$ be commutative rings, with $A$ semilocal and $B a$ finite faithful $A$-algebra. Then $B$ is a free $A$-module of rank $n$ if $B \otimes_{A} B$ is a free $B$-module (under left action) of rank $n$.

Proof. Let $J$ be the Jacobson radical of $A$. We first suppose $J=0$. Then $A$ is a finite product of fields, and in this case the result is immediate. In general, we observe that, by going up and going down, $J B \cap A=J$, so $B / J B$ is faithful over $A / J$. Moreover, $B / J B \otimes_{A / J} B / J B=B / J B \otimes_{B}\left(B \otimes_{A} B\right)$ is free of rank $n$ over $B \mid J B$, and so by the case already considered, $B \mid J B$ is a free $A / J$-module of rank $n$. Choose $x_{1}, \cdots, x_{n}$ in $B$ such that their images form a basis of $B / J B$ over $A / J$. By Nakayama's lemma, the $x_{i}$ span $B$. Moreover, the $1 \otimes x_{i}$ then span $B \otimes{ }_{A} B$; since the latter is free of rank $n$ they are a basis. But any relation of linear dependence on the $x_{i}$ over $A$ gives a relation on the $1 \otimes x_{i}$ over $B$, and hence must be trivial. Thus the $x_{i}$ are free generators of $B$ over $A$.

THEOREM. Let $G \rightarrow H$ be an epimorphism in $k$-FCGS with kernel $L$. Then $G \rightarrow H$ is free of rank [L:1].

COROLlaRY. $k$-FCGS is an abelian category.

Proof. Let $p: G \rightarrow H$ be an epimorphism with kernel $L$. Let $C=$ Coker $(\operatorname{ker}(p))$. By the definition of $C, p$ factors as $G \rightarrow C \rightarrow H$ where both maps are epimorphisins. Since $L$ is also the kernel of $G \rightarrow C$, the rank of $A G$ as an $A C$ module is [L:1], which is the same as the rank of $A G$ as an $A H$ module. Thus the inclusion $A H \rightarrow A C$ is an isomorphism and so $G \rightarrow H$ is a cokernel. Since the category is self dual, every monomorphism is a kernel.

(The corollary also follows from the theorem and the fact that faithfully flat morphisms are effective epimorphisms.)

We note that the theorem is a special case of the theorem that passage to the quotient by a finite flat equivalence relation on an affine scheme is faithfully flat [2, Theorem 4.1], and our approach is closely related to the proof of that theorem. Finally, it is the authors' belief that the mixture of cogroup and ring properties in the theory of Hopf algebras can make the subject difficult for nonspecialists; therefore it is often convenient to separate the properties (as we have tried to do here) by dealing with the functor of points, so ordinary group theory may be used as far as possible. 


\section{REFERENCES}

1. P. Freyd, Abelian categories. An introduction to the theory of functors, Harper's Series in Modern Math., Harper and Row, New York, 1964. MR 29 \#3517.

2. P. Gabriel, Construction de préschémas quotient, Schémas en Groupes (Sém. Géométrie Algébrique, Inst. Hautes Études Sci., 1963/64), fasc. 2a, exposé 5, Inst. Hautes Études Sci., Paris, 1965. MR 41 \#1749.

3. F. Oort and J. R. Strooker, The category of finite bialgebras over a field, Nederl. Akad. Wetensch. Proc. Ser. A 70=Indag. Math. 29 (1967), 163-169. MR 35 \#1599.

4. M. E. Sweedler, Hopf algebras, Math. Lecture Note Series, Benjamin, New York, 1969. MR 40 \#5705.

Department of Mathematics, Graduate Center, City University of New YORK, NEW YORK, NEW YORK 10036

Department of Mathematics, Columbia University, New York, New York 10027 (Current address of Andy R. Magid)

Current address (Raymond T. Hoobler): Department of Mathematics, Rice University, Houston, Texas 77001 